\title{
Mechanoenergetic Estimation of Multiple Cross-Bridge Steps per ATP in a Beating Heart
}

\author{
Hiroyuki SUGA \\ National Cardiovascular Center Research Institute, Suita, 565-8565 Japan
}

\begin{abstract}
The efficiency from the ventricular $\mathrm{O}_{2}$ consumption $\left(\mathrm{VO}_{2}\right)$ to the total mechanical energy (TME) generated by ventricular contraction has proved relatively constant at $\sim 35 \%$, independent of the loading and contractile conditions in a canine heart. TME is the sum of the external mechanical work for ejecting a stroke volume against the afterload and of the mechanical potential energy for developing ventricular pressure in each beat. The $\sim 35 \% \mathrm{VO}_{2}$-to-TME efficiency indicates an also constant $\sim 60 \%$ ATP-to-TME efficiency in a beating heart, based on the nominal $\sim 60 \% \mathrm{VO}_{2}$-to-ATP efficiency in the myocardial oxidative phosphorylation. I newly attempted to explain the load-independent $\sim 60 \%$ ATP-to-TME efficiency by the recently reported $\sim 7-10 \mathrm{~nm}$ unitary step size and $\sim 0.8-1.5 \mathrm{pN}$ unitary force of a
\end{abstract}

cross-bridge (CB) at the molecular level in in vitro motility assays. This single $\mathrm{CB}$ behavior suggests that its unitary cycle could generate a mechanical energy of $\sim 0.6-1.5 \times 10^{-20} \mathrm{~J}$ at most. From the nominal free energy of $\sim 10 \times 10^{-20} \mathrm{~J}$ per ATP, the efficiency from one ATP to the CB unitary cycle would then be $\sim 6-15 \%$. This low efficiency is only $\sim 1 / 10-1 / 4$ of the $\sim 60 \%$ ATP-toTME efficiency at the heart level. This discrepancy suggests that each $C B$ would repeat the unitary cycle at least $\sim 4-10$ times per ATP to achieve the high constant ATP-to-TME efficiency in a beating heart. This seems to represent a considerable mechanoenergetic advantage of the heart at the integrative heart level as compared to the molecular CB level. [The Japanese Journal of Physiology 54: 103-108, 2004]

Key words: myocardium, mechanics, energetics, unitary cycle, sliding filament.

$\mathrm{T}_{\mathrm{h}}$ The efficiency from the ventricular $\mathrm{O}_{2}$ consumption $\left(V \mathrm{O}_{2}\right)$ to the total mechanical energy (TME) generated by ventricular contraction has proved to be relatively constant at $\sim 35 \%$, independent of the loading and contractile conditions in a beating canine heart [1-6]. This suggests a high constant efficiency of $\sim 60 \%$ from ATP to TME in a beating heart $[5,6]$, based on the $\sim 60 \%$ efficiency of the myocardial oxidative phosphorylation to generate ATP from $V_{\mathrm{O}_{2}}$ at the nominal 3P:O atomic stoichiometry (i.e., $\sim 0.35=$ $\sim 0.60 \times \sim 0.60$ ) [5-7]. The load-independence of this ATP-to-TME efficiency has been supported by other investigators measuring heat as the energy output [7, 8]. Furthermore, this organ-level mechanoenergetics framework established first in the dog heart has been confirmed in the heart preparations of different mammalian species such as the rabbit and rat $[9,10]$.

At the molecular level, recent in vitro motility as- says have shown that a cross-bridge (CB) made of myocardial actin and myosin molecules produces per an ATP molecule a $\sim 7-10 \mathrm{~nm}$ unitary step under no load and a $\sim 0.8-1.5 \mathrm{pN}$ unitary force under no shortening for both V1 and V3 myosin isoforms of the rat and rabbit $[11,12]$. Their product, i.e., step size times force, suggests that the CB unitary cycle per ATP could generate a mechanical energy of at most $\sim 0.6-1.5 \times 10^{-20} \mathrm{~J}$. Based on the nominal free energy of $\sim 10 \times 10^{-20} \mathrm{~J}$ per ATP [7], the efficiency from one ATP to the CB unitary cycle would then be $\sim 6-15 \%$. This efficiency is only $\sim 1 / 10-1 / 4$ of the $\sim 60 \%$ ATPto-TME efficiency in a beating heart.

In the present review, I considered theoretically on these already reported myocardial mechanoenergetics at both the organ level and the molecular level. Based on this consideration, I could estimate that each $\mathrm{CB}$ would repeat its unitary cycle several to a few dozen

Received on January 9, 2004; accepted on January 19, 2004

Correspondence should be addressed to: Hiroyuki Suga, Research Institute, National Cardiovascular Center, 5-7-1 Fujishirodai, Suita, 565-8565 Japan. Tel: +81-6-6833-5211, Fax: +81-6-6833-1421, E-mail: hsuga@ri.ncvc.go.jp 
times per ATP to achieve the high constant ATP-toTME efficiency in a beating heart. This seems to represent a considerable mechanoenergetic advantage of the heart at the integrative level in comparison to the elemental level.

\section{Background}

In cardiac mechanoenergetics, I proposed for the first time a specific area (systolic pressure-volume area, PVA) as a measure of TME generated by each left ventricular (LV) contraction in the pressure-volume $(P-V)$ diagram (Fig. 1A) [5, 6, 13]. PVA consists of the $P-V$ area representing the external mechanical work (EW) and that representing the mechanical potential energy (PE) generated in the ventricular wall. Namely, TME is the sum of EW and PE (Fig. 1A) [5, $6,13]$.

We have then found that TME measured by the PVA linearly correlates with $\mathrm{VO}_{2}$ in canine LVs (Fig. 1B) $[5,6,13]$. The slope of the $\mathrm{VO}_{2}$-to-TME relation above the sum of the basal metabolism and excitationcontraction coupling $\mathrm{VO}_{2}$ components is constant regardless of the LV loading and contractile conditions $[5,6,13]$. This mechanoenergetic framework reasonably matches with the already established time-varying elastance model of the LV (Fig. 1C) [5, 14].

This unique cardiac mechanoenergetics is consistent with the cardiac Fenn effect $[1,3,5,8]$, which is significantly different from the original Fenn effect established in the skeletal muscle $[7,15]$. The heart requires much less shortening heat or energy than the skeletal muscle [5, 7]. This difference leads to a higher mechanical efficiency in shortening and ejecting contractions of the heart $[5,7]$. Moreover, the time-varying elastance model I established in the heart in the 1970s does not hold for the skeletal muscle [7]. In the skeletal muscle, the so-called cocked spring model with an instantly rising elastance was discarded because its energetic prediction did not match the skeletal muscle Fenn effect [15]. In contrast, the energetic prediction of the time-varying elastance model of the heart reasonably matches the cardiac Fenn effect [1, 3, 4, 7].

The linear relationship between $V \mathrm{O}_{2}$ and TME (Fig. 1B) above the $\mathrm{VO}_{2}$ components for both the basal metabolism and the excitation-contraction coupling indicates a load- and contractility-independent constant $\mathrm{O}_{2}$ cost of TME $[5,6]$. Its reciprocal indicates a constant contractile efficiency of $\sim 35 \%$ (Fig. 1B) $[5,6]$. Note that this efficiency is different from and greater than the conventional mechanical efficiency from $\mathrm{VO}_{2}$ to the external mechanical work which is part of TME
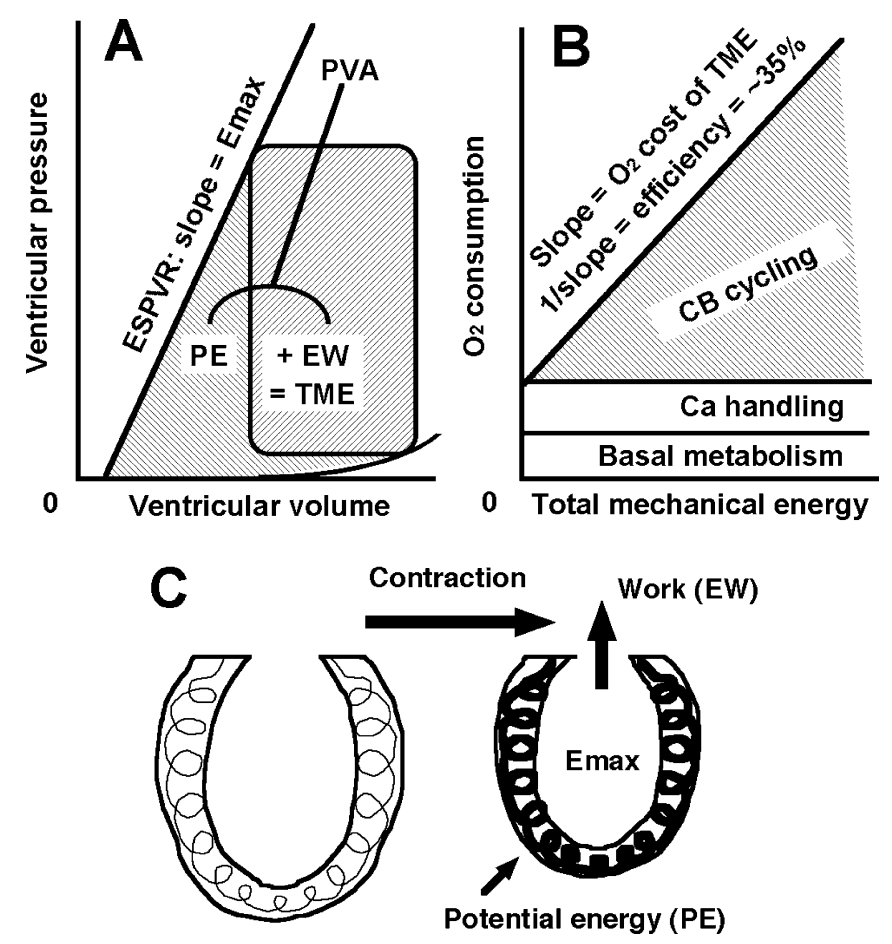

Fig. 1. Cardiac mechanoenergetics framework at the heart level. A: Total mechanical energy (TME) corresponds to the specific area (shaded, designated as pressure-volume area, and abbreviated as PVA) under the end-systolic pressure-volume $(P-V)$ relation (ESPVR) in the left ventricular $P-V$ diagram. TME consists of external mechanical work $(E W)$ and mechanical potential energy (PE). B: Experimentally established a linear relationship between the left ventricular $\mathrm{O}_{2}$ consumption and TME regardless of its loading conditions, whether isovolumic or ejecting at a constant contractile condition. The slope of the diagonal linear relation indicates $\mathrm{O}_{2}$ cost of TME, and its reciprocal indicates the contractile efficiency from $\mathrm{O}_{2}$ to TME. The increasing $\mathrm{O}_{2}$ consumption with TME is for cross-bridge (CB) cycling (shaded). The other part of $\mathrm{O}_{2}$ consumption independent of TME is used for the non-mechanical activities such as basal metabolism and calcium $(\mathrm{Ca})$ handling for excitationcontraction coupling. Changes in contractile state $\left(E_{\max }\right)$ affect only the $\mathrm{O}_{2}$ consumption for Ca handling and then the elevation of the linear relation between left ventricular $\mathrm{O}_{2}$ consumption and TME. C: The concept of TME was deduced from the time-varying elastance model of the ventricle. When the chamber increases its volume elastance to its maximum $E_{\max }$ by contraction, the increasing elastance generates not only EW to the afterload when ejection occurs, but also PE within the stiffening wall.

(Fig. 1A) [5, 6]. Therefore, although the mechanical efficiency varies between 0 and $\sim 30 \%$ depending on the fraction of the mechanical work in TME, the contractile efficiency remains constant at $\sim 35 \%$ [5]. Since the oxidative phosphorylation in myocardium has a nominal 3P:O atomic stoichiometry equivalent to a chemical efficiency of $\sim 60 \%$, the $\sim 35 \%$ efficiency from $\mathrm{VO}_{2}$ to TME means a constant efficiency 
of $\sim 60 \%$ from ATP to TME (Fig. 2A) (i.e., $\sim 0.6=$ $\sim 0.35 / \sim 0.6$ ) [5-7].

At the molecular level, the in vitro motility assay of skeletal muscle actin-myosin interactions has shown that a load-free CB could slide over multiple unitary steps per ATP [16-19]. A tight coupling between ATP and $\mathrm{CB}$ sliding, though, is not fully negated for skeletal muscle CBs [20, 21]. Apart from the skeletal muscle, Sugiura et al. discovered that each attached CB produces a unitary step sliding of a gaussian peak of $\sim 10 \mathrm{~nm}$ under no load and a unitary force of a mean peak of $\sim 1.5 \mathrm{pN}$ under no sliding, using rat myocardial actin and myosin (V1 and V3 isoforms) molecules in an in vitro motility assay [11]. Palmiter et al. reported a unitary step size of $\sim 7 \mathrm{~nm}$ and a unitary force of $\sim 0.8 \mathrm{pN}$, using rabbit actin and myosin (V1 and V3 isoforms) molecules [12]. These step sizes are largely comparable to the myosin-head length and seem reasonable if the CB sliding is driven by the tilting of a myosin head attached to an actin filament (Fig. 2B). These unitary values fall in the lower range of the step size $(\sim 6-20 \mathrm{~nm})$ and are smaller than or comparable to the force $(\sim 1.5-6 \mathrm{pN})$ of skeletal muscle $\mathrm{CBs}$ in the literature [16-21]. However, unlike a skeletal muscle $\mathrm{CB}$, no group has yet reported evidence of multiple steps of a cardiac CB cycling per ATP.

Knowing the multiple steps of a skeletal muscle CB per ATP reported by Yanagida et al. [19], we have theoretically indicated the possibility of multiple steps of each CB per ATP to account for the cardiac Fenn effect [22] by use of the Huxley-type CB model [23]. However, we could not relate the resultant values of the multiple steps of CBs [22] with the high constant contractile efficiency at the heart level because unitary step size and force data of a cardiac CB were not available before the work of Sugiura et al. [11].

\section{Theoretical Considerations}

Knowing the unitary behavior of a cardiac CB [11, 12], I again wondered whether it can account for the load-independent high constant efficiency of $\sim 60 \%$ from ATP to TME in a beating heart, and if it can account for it, how this is possible. To this end, I calculated first in the present study the CB unitary energetics, using the CB unitary mechanics reported by Sugiura et al. [11]. From the unitary step size of $\sim 10 \mathrm{~nm}$
A
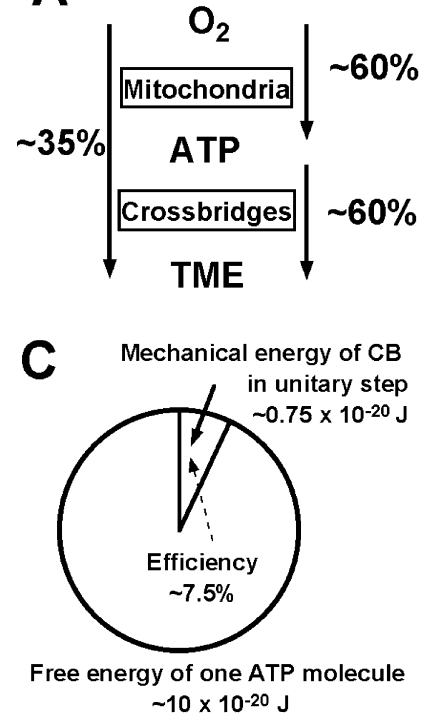

$\sim 10 \times 10^{-20} \mathrm{~J}$
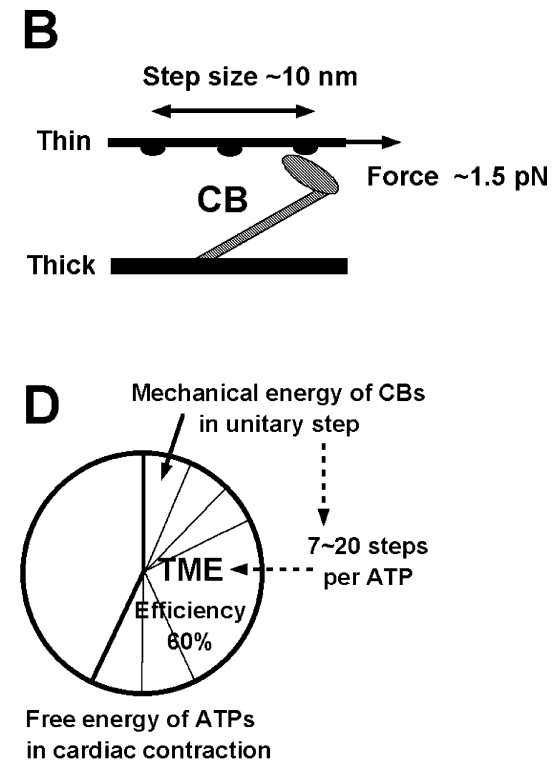
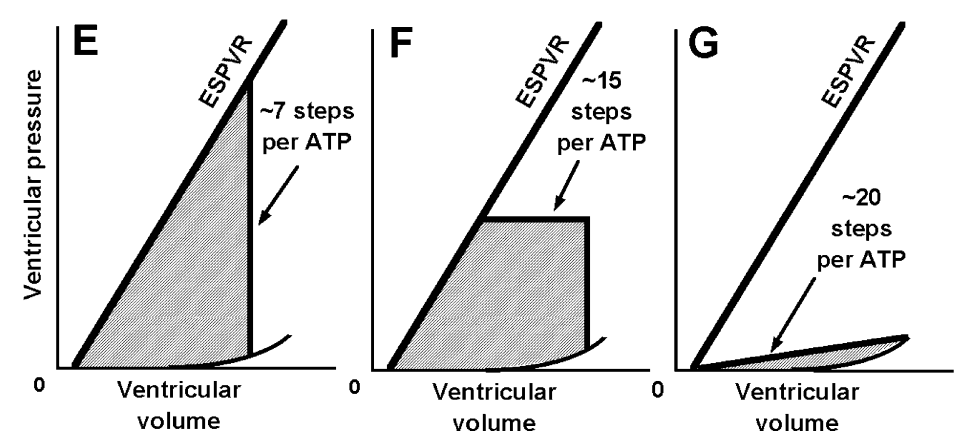

Fig. 2. Unitary cross-bridge cycling per ATP at the molecular and organ levels. A: Efficiencies of energy conversions from $\mathrm{O}_{2}$ to total mechanical energy (TME) via ATP in myocardium. B: Unitary step size and force of the attached cross bridge $(\mathrm{CB})$ in the myosin-swinging and filamentsliding model. The unitary values were cited from Sugiura et al. [11]. C: Efficiency of mechanical energy generated by a unitary step of a CB from the free energy of an ATP molecule at the molecular level. D: Efficiency of TME generated by multiple $(\sim 7-20)$ unitary steps of a CB from the free energy of ATP in cardiac contraction. E, F, and $G$ : Average numbers of unitary steps of a CB per ATP estimated in a ventricular contraction under three different loading conditions: isovolumic contraction (E); moderately afterloaded, isobarically ejecting contraction (F); and preloaded but almost freely ejecting contraction $(\mathbf{G})$. The numbers of the unitary steps in these panels will increase reciprocally with the decreases in the unitary step size and force of a CB per ATP from Sugiura et al.'s data [11] to Palmiter et al.'s data [12]. 
and the unitary force of $\sim 1.5 \mathrm{pN}$ of a single CB [11], the unitary mechanical energy generated by a single $\mathrm{CB}$ cycle would be at most their product, i.e., $\sim 15 \mathrm{pN} \mathrm{nm}=\sim 1.5 \times 10^{-20} \mathrm{~J}(\mathrm{~N} \mathrm{~m}=\mathrm{J}$ and $100 \mathrm{pN} \mathrm{nm}=$ $10 \times 10^{-20} \mathrm{~J}$ ) in the swinging CB model (Fig. 2B).

However, the force of a $\mathrm{CB}$ attached to an actin molecule will reasonably decrease with sliding or shortening from the maximal reach $(\sim 10 \mathrm{~nm})$ of the actin-attached myosin head in the attached CB [23]. If the unitary force of an attached $\mathrm{CB}$ is assumed to decrease linearly with its shortening, the unitary mechanical energy generated by the single $\mathrm{CB}$ cycle could be predicted to decrease to half of $\sim 1.5 \times$ $10^{-20} \mathrm{~J}$, i.e., $\sim 0.75 \times 10^{-20} \mathrm{~J}$ (Fig. $2 \mathrm{C}$ ).

Since ATP has nominally a free energy of $57 \mathrm{~kJ} / \mathrm{mol}$ in myocardium [7], an ATP molecule has a free energy of 9.5 (or $\sim 10) \times 10^{-20} \mathrm{~J}$ as $57 \mathrm{~kJ} / \mathrm{mol}$ divided by Avogadro's number of $6.02 \times 10^{23} \mathrm{molecules} / \mathrm{mol}$. From the mechanical energy of the above mentioned $\sim 0.75 \times 10^{-20} \mathrm{~J}$ generated by a unitary $\mathrm{CB}$ cycle and the free energy of the above-mentioned $\sim 10 \times 10^{-20} \mathrm{~J}$ of an ATP molecule, we can calculate the chemomechanical efficiency from one ATP to a CB unitary cycle to be $\sim 7.5 \%$ (Fig. 2 C). This is only $\sim 1 / 8$ of the mechanical efficiency of $\sim 60 \%$ from ATP to TME in the ventricle.

To solve this $\sim 8$ times discrepant efficiencies between the organ and molecular levels, it seems quite reasonable to imagine that each attached CB cycles $\sim 8$ times on average per ATP, not merely once, to convert the chemical energy of ATP to TME at the high overall efficiency of $\sim 60 \%$ in a beating heart (Fig. 2D).

Myocardial sarcomeres usually shorten even in the isometric contraction by stretching the series elasticity that is several times more compliant than that of the skeletal muscle [24]. Moreover, myocardium per se normally shortens more or less in the normally asynchronously contracting ventricular wall, even without ejection in an isovolumic contraction. These mechanical properties of the myocardium should allow the attached CBs on average to slide even when the myocardium contracts isometrically and the ventricle contracts isovolumically (Fig. 2E).

When the series elasticity is stretched by $\sim 7 \%$ at the peak of isometric myocardial contraction [24], it allows a half-sarcomere of $\sim 1 \mu \mathrm{m}$ length to shorten by $\sim 70 \mathrm{~nm}$, which is 7 times the CB unitary step of $\sim 10 \mathrm{~nm}$. This suggests that the attached CB must slide over $\sim 7$ unitary steps on average to develop the high peak ventricular pressure in an isovolumic contraction (Fig. 2E).

Furthermore, during ejection with a typical normal ejection fraction of $\sim 70 \%$ against a moderate afterload, myocardium and then half-sarcomeres of $\sim 1 \mu \mathrm{m}$ length would shorten by $\sim 15 \%$ on average, though varying among different layers of the ventricular wall. This shortening corresponds to $\sim 150 \mathrm{~nm}$ and thus to $\sim 15$ unitary steps of a CB (Fig. 2F).

As the force generated by each sliding $\mathrm{CB}$ decreases with its sliding distance or shortening velocity, the load-independent $\sim 60 \%$ efficiency from ATP to TME suggests that the number of unitary steps of a CB per ATP could increase considerably despite the constant $\sim 10 \mathrm{~nm}$ unitary steps of a CB [11]. If the number of unitary steps of a CB per ATP were always one despite the changing load, the load-independent constant efficiency from ATP to TME could not be maintained. Such a load-dependent efficiency from ATP to TME contradicts the reality [5]; therefore, the multiple unitary steps of a CB on average per ATP are reasonable even in shortening contractions. The multiple unitary steps per ATP would reasonably hold because even the ejecting contraction requires $\mathrm{VO}_{2}$ proportional to TME without requiring extra $\mathrm{VO}_{2}$ for ejection and muscle shortening $[4,5]$ in contrast to skeletal muscles [4]. This is the essential difference of the cardiac Fenn effect from the skeletal muscle Fenn effect $[7,15]$.

As an extreme case, the pre-loaded ventricle can eject its entire volume against a small afterload with almost no extra $\mathrm{VO}_{2}$ for a practically zero TME above the $\mathrm{VO}_{2}$ of a non-preloaded contraction [2, 5]. In this case, the half-sarcomere would shorten reasonably from $\sim 1$ to $\sim 0.8 \mu \mathrm{m}$ by $\sim 200 \mathrm{~nm}$, corresponding to $\sim 20$ unitary steps of a CB per ATP (Fig. $2 \mathrm{G}$ ).

These $\sim 7$ to $\sim 20$ multiple unitary steps on average of each CB per ATP (Fig. 2E, F, and G) may appear contradictory to our previous calculation that each attached CB hydrolyzes ATP once or a little more on average in one contraction of the heart $[6,25,26]$. It was calculated from a reasonably maximum TME of $4,000 \mathrm{mmHg} \mathrm{ml}=\sim 0.53 \mathrm{~J}$ per $100 \mathrm{~g} \mathrm{LV}$ generated by all the $\sim 15 \mu \mathrm{mol} \mathrm{CBs}$ per $100 \mathrm{~g} \mathrm{LV}[6,25,26]$. This number of $\mathrm{CBs}$ is reasonably presumed from a representative myocardial concentration of myosin molecules of $\sim 7.5 \mu \mathrm{mol}$ per $100 \mathrm{~g} \mathrm{LV}$, and the two heavy chains attachable to actin per myosin molecule. The above TME divided by the total number of CBs yields TME of $\sim 6 \times 10^{-20} \mathrm{~J}$ per CB. Since each ATP has $\sim 10 \times 10^{-20} \mathrm{~J}$, as already mentioned, the $60 \%$ efficiency of the above TME per ATP leads to $\sim 6 \times$ $10^{-20} \mathrm{~J}$. This is comparable to the above-mentioned TME of $\sim 6 \times 10^{-20} \mathrm{~J}$ per CB. The coincidence of these two energy values always holds regardless of the loading and contractile conditions because of the 
load-independent constancy of the contractile efficiency $[5,6]$. This implies that each once-attached CB hydrolyzes ATP once on average regardless of the number of its multiple unitary steps during any cardiac contraction under a variety of loading and contractile conditions.

In these calculations, I adopted the unitary step size and force values reported by Sugiura et al. [11]. However, if I use those reported by Palmiter et al. [12], $\sim 2.5$ times more unitary steps of a CB per ATP would be required to maintain the constant $\sim 60 \%$ ATP-toTME efficiency.

Based on these results, I would propose the possibility that each attached CB slides over several to a few dozen unitary steps by gradually converting the ATP free energy to TME in each of the multiple unitary steps (Fig. 2D-G). This possibility seems to represent a mechanoenergetic advantage of the heart at an integrative level over the mechanoenergetics of individual $\mathrm{CB}$ molecules at an elemental level. This possibility is the first reasonably quantitative one to bridge between the integrative (or organ or global) and reductive (or molecular or elemental) experimental findings on cardiac mechanoenergetics. I recently presented this aspect of cardiac contraction in a symposium $[27,28]$.

This work was partly supported by a Cardiovascular Diseases Research Grant (14A-1) from the Ministry of Health, Labor and Welfare and Grants-in-Aid for Scientific Research $(13878192,16659057)$ from the Ministry of Education, Culture, Sports, Science and Technology, Japan.

\section{REFERENCES}

1. Nozawa T, Yasumura $Y$, Futaki S, Tanaka N, and Suga $\mathrm{H}$ : The linear relation between oxygen consumption and pressure-volume area can be reconciled with the Fenn effect in dog left ventricle. Circ Res 65: 13801389, 1989

2. Yasumura $Y$, Nozawa T, Futaki S, Tanaka N, and Suga $\mathrm{H}$ : Minor preload dependence of $\mathrm{O}_{2}$ consumption of unloaded contraction in dog heart. Am J Physiol 256: H1289-H1294, 1989

3. Hata K, Goto Y, and Suga H: External mechanical work during relaxation period does not affect myocardial oxygen consumption. Am J Physiol 261: H1778H1784, 1991

4. Kawaguchi O, Goto $Y$, Futaki S, Ohgoshi $Y$, Yaku $H$, Hata K, Takasago T, Saeki A, and Suga H: Ejecting deactivation does not affect $\mathrm{O}_{2}$ consumption-pressurevolume area relation in dog hearts. Am J Physiol 265: H934-H942, 1993

5. Suga H: Ventricular energetics. Physiol Rev 70: 247277, 1990

6. Suga H: Global cardiac function: mechano-energetico- informatics. J Biomech 36: 713-720, 2003

7. Gibbs CL and Barclay CJ: Efficiency of skeletal and cardiac muscle. Adv Exp Med Biol 453: 527-536

8. Mast $F$ and Elzinga $G$ : Heat released during relaxation equals force-length area in isometric contractions of rabbit papillary muscle. Circ Res 67: 893-901, 1990

9. Goto Y, Slinker BK, and LeWinter MM: Similar normalized Emax and $\mathrm{O}_{2}$ consumption-pressure-volume area relation in rabbit and dog. Am J Physiol 255: H366H374, 1988

10. Hata $Y$, Sakamoto T, Hosogi S, Ohe T, Suga H, Takaki M: Linear $\mathrm{O}_{2}$ use-pressure-volume area relation from curved end-systolic pressure-volume relation of the blood-perfused rat left ventricle. Jpn J Physiol 48: 197-204, 1998

11. Sugiura S, Kobayakawa N, Fujita H, Yamashita $H$, Momomura S, Chaen S, Omata M, and Sugi H: Comparison of unitary displacements and forces between 2 cardiac myosin isoforms by the optical trap technique: molecular basis for cardiac adaptation. Circ Res 82: 1029-1034, 1998

12. Palmiter KA, Tyska MJ, Dupuis DE, Alpert NR, and Warshaw DM: Kinetic differences at the single molecule level account for the functional diversity of rabbit cardiac myosin isoforms. J Physiol (Lond) 519: 669678, 1999

13. Suga $\mathrm{H}$ : Total mechanical energy of a ventricle model and cardiac oxygen consumption. Am J Physiol 236: H498-H505, 1979

14. Suga $H$, Sagawa $K$, and Shoukas AA: Load independence of the instantaneous pressure-volume ratio of the canine left ventricle and effects of epinephrine and heart rate on the ratio. Circ Res 32: 314-322, 1973

15. Fenn WO: The relation between the work performed and the energy liberated in muscle contraction. J Physiol (Lond) 58: 174-254, 1924

16. De Winkel ME, Blange T, and Treijtel BW: Viscoelastic properties of cross bridges in cardiac muscle. Am J Physiol 268: H987-H998, 1995

17. Guilford WH, Dupuis DE, Kennedy G, Wu J, Patlak JB, and Warshaw DM: Smooth muscle and skeletal muscle myosins produce similar unitary forces and displacements in the laser trap. Biophys J 72: 1006-1021, 1997

18. Uyeda TQ, Kron SJ, and Spudich JA: Myosin step size. Estimation from slow sliding movement of actin over low densities of heavy meromyosin. J Mol Biol 214: 699-710, 1990

19. Yanagida T, Arata T, and Oosawa F: Sliding distance of actin filament induced by a myosin crossbridge during one ATP hydrolysis cycle. Nature 316: 366-369, 1985

20. Finer JT, Simmons RM, and Spudich JA: Single myosin molecule mechanics: piconewton forces and nanometre steps. Nature 368: 113-119, 1994

21. Toyoshima YY, Kron SJ, and Spudich JA: The myosin step size: measurement of the unit displacement per ATP hydrolyzed in an in vitro assay. Proc Natl Acad Sci USA 87: 7130-7134, 1990

22. Taylor TW, Goto $Y$, and Suga H: Variable cross-bridge cycling-ATP coupling accounts for cardiac mechanoenergetics. Am J Physiol 264: H994-H1004, 1993

23. Huxley AF: Muscle structure and theories of contraction. J Prog Biophys Biophys Chem 7: 255-318, 1957 


\section{H. SUGA}

24. Suga H: Systolic quick releases of nonexcised bloodperfused canine papillary muscle. Am J Physiol 238: H153-H155, 1980

25. Suga H, Goto Y, Kawaguchi O, Hata K, Takasago T, Saeki A, and Taylor TW: Ventricular perspective on efficiency. In: Myocardial Optimization and Efficiency, Evolutionary Aspects and Philosophy of Science Considerations, ed. Burkhoff D et al., Springer-Verlag, New York 1994

26. Suga H, Goto Y, Kawaguchi O, Hata K, Takasago T, Saeki A, and Taylor TW: Ventricular perspective on effi- ciency. Basic Res Cardiol 88 (Suppl 2): 43-65, 1993

27. Suga $H$ : Cardiac mechanoenergetic physiome: from Emax and PVA to calcium and crossbridge. First International Symposium of Cardiovascular Physiome-Integrative Comprehension from Molecule to Organ, December 5-7, 2003

28. Suga $\mathrm{H}$ : Mysterious beauty of beating heart: cardiac mechano-energetico-informatics. Symposium on Mysteries about the Sliding Filament Mechanism in Muscle Contraction: Fifty Years after its Proposal, Tokyo, March 7-10, 2004 